\title{
Investigación
}

\section{La actual transición tecnológica y su relación con la gerencia empresarial}

\author{
The current technological transition \\ and its relationship with business \\ management
}

\author{
Diana Zoleidy Jaramillo Sierra ${ }^{1}$
}

- Recepción: 18/02/2020 • Aprobación: 14/04/2020 • Publicación: 15/07/2020

Para citar este artículo

Jaramillo Sierra, D. Z. (2020). La actual transición tecnológica y su relación con la gerencia empresarial. Dos mil tres mil, 22, e222.

https://doi.org/10.35707/dostresmil/22222
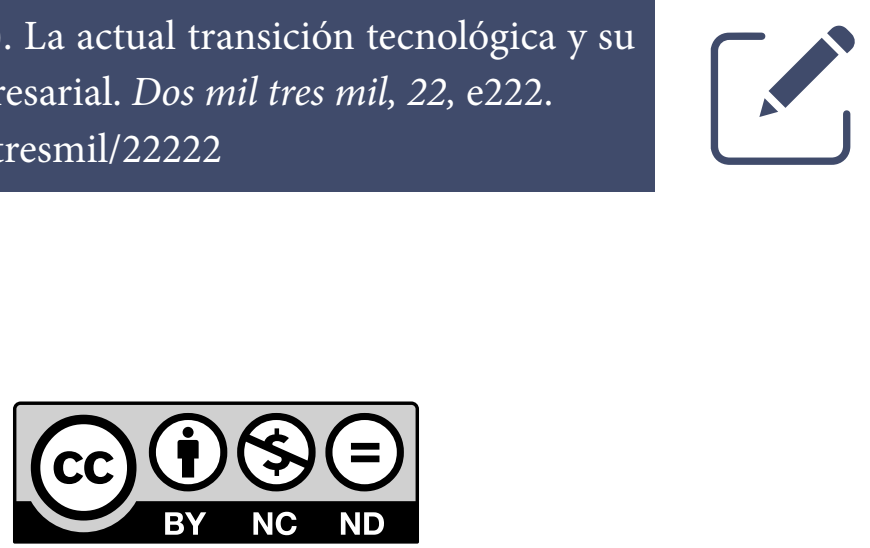

${ }^{1}$ Fundación Universitaria San Martín, Colombia. ORCID: 0000-0001-9315-4727. Correo electrónico: diana.jaramillos@sanmartin. edu.co 
Resumen. La finalidad de este artículo es abordar la relación que propone la transición de la cuarta revolución industrial y la gerencia empresarial, a partir de una noción primaria sobre los conceptos de ambas variables y la evolución que han tenido en los últimos años. Sumado a esto, se presentan algunas comparaciones entre la gerencia tradicional y la gerencia contemporánea, la inserción de la cuarta revolución industrial en el rol gerencial, así como las ventajas y desventajas que esta trae para las empresas de hoy. La metodología que se utiliza es la revisión bibliográfica a partir de la exploración teórica de algunos autores, bajo un enfoque descriptivo que caracterice la relación que proponen las variables abordadas y que finalmente aporten a la construcción de algunas apreciaciones sobre este desafío tecnológico en la gerencia empresarial actual.

Abstract. This article aims to address the relationship proposed by the transition of the Fourth Industrial Revolution and Business Management, from an elementary notion regarding the concepts of both variables and the evolution they have had in the latest years. In addition to this, some comparisons are presented between Traditional Management and Contemporary Management, the insertion of the Fourth Industrial Revolution in the managerial role, as well as the advantages and disadvantages that it brings for current companies. The methodology used is the bibliographic review based on the theoretical exploration of some authors; using a descriptive approach that characterizes the relationship proposed by the variables addressed, and that ultimately contributes to the construction of some appreciations of this technological challenge in today's Business Management.

Palabras claves

Gerencia, revolución, tecnología, administrar.

Key words

Management, revolution, technology, manage. 


\section{Conceptos teóricos preliminares: Gerencia}

El término gerencia ha sido ampliamente difundido en las ciencias administrativas, toda vez que su concepto varía de acuerdo con la opinión de los autores sobre el tema; en este ejercicio interesa comprender de qué manera se construye el concepto de gerencia en el contexto empresarial. Al respecto, Drucker (1957) señala: "La gerencia es el órgano de la sociedad encargado específicamente de hacer productivos los recursos, es decir, responsable del progreso económico organizado" (p. 15).

Sin embargo, un concepto de orden más organizacional lo plantea Polo, al afirmar que:

La concepción actual de la gerencia en la organización se enfoca en prestar atención no solo a los factores de producción y mercado, sino en lograr la integración total de la Gestión Humana con la misión y visión de la organización. Es decir, que no pudiera pensarse la gerencia sin una organización de personas, que le sirva de soporte para alcanzar objetivos planteados por la misma. (Polo, 2011, p. 66).

Otra perspectiva interesante la plantea Álvarez (2013), al mencionar que "el modelo de gerencia es una referencia o guía de administración de una empresa, sobre este modelo se fundamentan las acciones y decisiones que se toman ante una situación determinada para alcanzar los objetivos de una organización" (p. 4). Este referente enmarca la gerencia en el componente del direccionamiento estratégico del cual emanan unas políticas que orientan el desempeño de la empresa.

Los anteriores conceptos abarcan respectivamente palabras claves como recursos, estrategias y acciones. Al considerar estos términos, se puede esbozar una definición sobre gerencia como un rol organizacional, encargado del ejercicio de la gestión de los recursos, las estrategias y las acciones para el eficiente desempeño de la empresa.

\section{Evolución de la gerencia. Una mirada al pasado}

Históricamente, los primeros procesos gerenciales se llevaron a cabo en las mismas organizaciones incipientes que se formaron para dar un orden a la sociedad. Posterior a ello, proyectos eclesiásticos y militares debieron ser gerenciados para cumplir algún tipo de objetivo. Al respecto, Ibáñez y Castillo afirman lo siguiente:

Para el siglo xv, Venecia se convierte en el foco del intercambio comercial, comenzando así el desarrollo de las empresas que conocemos actualmente [...] En este ámbito se dará énfasis dentro del campo industrial y empresarial, para lo cual se han de considerar apreciaciones de científicos como Adam Smith, Carlos Marx, Frederick Taylor, Henry Fayol, Elton Mayo, Max Weber, entre otros; quienes desde el punto de vista gerencial de los sistemas organizacionales han otorgado a lo largo de la historia, aportes de principios filosóficos y teorías de funcionamiento organizativo. (Ibáñez \& Castillo, 2010, p. 69). 
Durante los años 60 y 70, se da paso al término "estrategia" y con este se enmarca al gerente como aquel que tiene un pensamiento a largo plazo. Al respecto, Sallenave menciona:

En los años 60 y 70, fue la estrategia. Entonces los asesores de la organización de los años cincuenta cambiaron de nombre, pasaron a llamarse asesores en planificación estratégica. Su idea era que la empresa que tuviera una estrategia superior a la de sus competidores, era la que tuviera éxito. (Sallenave, 1995, p. 1).

Según Milagros Villasmil:

El entorno de los años noventa ha cambiado significativamente. La fuerte tendencia a la globalización, con el consecuente reforzamiento de la interdependencia entre las economías y sistemas sociopolíticos que trae aparejado, ha introducido importantes elementos perceptibles de cambio respecto a décadas pasadas. (Villasmil, 2010, p. 141).

En tal sentido, aparecen términos asociados al quehacer empresarial como organización por redes de trabajo, matrices de gestión, cultura corporativa, gestión de la calidad, entre otros, y se comienza a observar un gerente con un liderazgo basado en aquello que sabe hacer y en cómo sirve a sus colaboradores. Podría decirse que esta década prepara el camino hacia la competitividad y el empoderamiento.

El siglo Xxi trajo consigo una serie de eventos que estremecieron la manera como las empresas se administraban. "La creciente inestabilidad en el entorno económico, la globalización de los mercados y la economía del conocimiento, son tres de las nuevas tendencias mundiales que en los albores del siglo xxi mayor impacto han tenido en la dirección empresarial" (Fracica, 2010, p. 56). Este nuevo milenio exige una actuación trascendental por parte de la gerencia, la cual debe poseer la suficiente inteligencia para evaluar oportunidades y amenazas propias de un entorno cuyos cambios se basen en el conocimiento.

$\mathrm{Al}$ tener en cuenta lo anterior, Crissien (2005) afirma: "La gerencia ha evolucionado como el hombre mismo, con cambios históricos de forma y de fondo, a partir de tres tipos y diferencias estructurales, dentro de las cuales están: el enfoque, la orientación y, finalmente, los mercados" (p. 61).

\section{Gerencia tradicional vs. Gerencia contemporánea}

Hasta este punto se ha abordado el concepto de gerencia desde un ángulo clásico o tradicional, en el que se registra de una manera metódica el orden de trabajo; predomina una visión borrosa sobre el entorno y el futuro, se mantienen procesos rígidos y cerrados, y los logros o metas alcanzadas son el resultado de la imposición directa sobre la fuerza de trabajo, haciendo de la estructura y la jerarquía una base piramidal de estricto control sobre las áreas de la empresa.

Sin embargo, en una realidad global y vertiginosamente cambiante como la actual, la concepción de gerencia contemporánea toma cada vez más importancia. Tendencias tan significativas como la disrupción digital, la robotización, modelos de negocios sustentables, conocimiento 
laboral y cambios demográficos, hacen que el ambiente competitivo se mida hoy por el desempeño gerencial de las empresas en todo el planeta. Al respecto, Drucker menciona:

A medida que la empresa pasa a ser una confederación o una agrupación, se hace cada vez más necesaria una alta gerencia separada, poderosa y responsable. Sus responsabilidades abarcarán la dirección, planeación, estrategia, valores y principios de la organización como un todo; su estructura y las relaciones entre los diversos miembros; sus alianzas, asociaciones y operaciones a riesgo compartido; y su investigación, diseño e innovación [...] Una tarea igualmente importante para la alta gerencia en la empresa de la sociedad futura será balancear sus tres dimensiones: como organización económica, como organización humana y como organización social, cada vez más importante. (Drucker, 2002, p. 54).

Desde esta perspectiva, se observa una gerencia moderna que no solo se enfoca en la empresa como tal, sino que se basa en formar y fortalecer al talento humano, se encauza en los cambios futuros y en los inmediatos, al lograr que tanto la empresa como el personal generen ganancias económicas sólidas, mejorando el beneficio mutuo, así como el social. La gerencia moderna no posee una base de planificación piramidal, se forja en una red de subsistemas cuyas estructuras son cada vez más planas y en la que las competencias y habilidades gerenciales son cada vez más blandas.

\section{Revoluciones industriales. Evolución y progreso}

A mediados del siglo XVIII, la economía en Europa dependía de las actividades agrícolas y artesanales. Procesos de comercialización escasos debido al pequeño tamaño de las urbes y al bajo crecimiento demográfico, enmarcaron este momento de la historia. Se hizo necesario mejorar las condiciones de vida, la sustitución del trabajo manual y la energía humana por el uso de máquinas, dando paso a la primera revolución industrial, representada por la maquinización. Desde el punto de vista gerencial, esta revolución originó un pensamiento administrativo basado en la administración científica, la estructura organizacional, las funciones dentro de la organización y las relaciones humanas.

Este cambio representó un considerable impacto en la sociedad a mediados del siglo xix. Esta nueva forma de ver la industria desencadenó una serie de avances en cuanto a la química, el petróleo y el acero; lo anterior, como una segunda fase de la primera revolución industrial, enmarcada por la electricidad. Esto hace que se produzcan cambios radicales en los transportes y comunicaciones. Surgen líneas de producción en masa, que exigían grandes volúmenes de energía a partir de combustibles fósiles que generaron crisis en el sector económico, el energético y el ambiental. Indiscutiblemente, los avances fueron tan notables, como la forma de gerenciar las grandes empresas, dando paso a una enmarañada jerarquía integrada por diversas áreas funcionales y un sin número de procesos y procedimientos mucho más complejos para dar soporte al gran tamaño de las industrias. 
Así las cosas, durante los años cincuenta, sesenta y setenta, los avances tecnológicos no cesaron de realizarse en ningún momento, dando lugar a la tercera revolución industrial, enmarcada en la informática. Esta trae consigo procesos tecnológicos computarizados y nuevas formas de comunicación a través del internet, lo cual cambia sustancialmente la forma de integrar el sistema productivo y en como las empresas llevan a cabo sus negocios. Se presenta, por consiguiente, la necesidad de implementar las Tecnologías de las Información y la Comunicación (TIC) como punto de partida de la competitividad por parte de una gerencia orientada a aspectos estratégicos como el liderazgo, la diferenciación de productos, el enfoque en nichos de mercado y el fortalecimiento de las relaciones con clientes y proveedores.

Todos estos adelantos que se iniciaron en la industria pasaron a mejorar la calidad de vida de las personas; es por eso que hoy no se concibe a un ciudadano que no esté relacionado en su quehacer diario con estas tecnologías. Ahora la humanidad se encuentra inmersa en una serie de cambios que están dando lugar a la cuarta revolución industrial. Esta sugiere organizaciones inteligentes, que apuntan a la inteligencia artificial, relacionada con hologramas, nanotecnología, los Big-Data, mega sistemas para almacenar datos; la nanotecnología que sugiere aparatos cada vez más pequeños y con mayor capacidad y la desfragmentación de la producción que implica la participación de varios países en la fabricación de productos, entre otros, que respaldan el modelo de una industria inteligente y que afectan considerablemente la forma en la que las personas se relacionan, se comunican, trabajan, etc. Al respecto, Falconi, et al., afirman lo siguiente:

Una organización inteligente con la determinación del éxito debería, por lo tanto, ser movida por un gerente que procure tener un equipo inteligente de empleados en un ambiente propicio, que pueda promover la innovación y el aprendizaje adicional. Depende de las empresas organizar cómo su equipo de empleados forma parte de las habilidades extraordinarias y es administrado por líderes competentes en dimensiones más amplias en esta era de la industria 4.0. (Falconi et al., 2018, s. p.).

$\mathrm{Al}$ atender a la anterior premisa, es evidente que los gerentes necesitan estar preparados para hacer frente a estos nuevos desafíos a partir de estrategias que beneficien a sus grupos de interés, en cuanto a la tecnología, el talento y la fuerza de trabajo, el ámbito social, el medio ambiente, y la misma gerencia.

\section{La cuarta revolución en las empresas de hoy. Ventajas y desventajas}

A continuación se presenta una tabla comparativa que permite identificar algunos de los beneficios y dificultades que trae para las empresas la tecnología 4.0, en relación a cinco variables: tecnología, fuerza de trabajo, el ámbito social, el medio ambiente y la gerencia. 
Tabla 1. Ventajas y desventajas de la tecnología 4.0

\begin{tabular}{|c|c|}
\hline Ventajas & Desventajas \\
\hline \multicolumn{2}{|l|}{ Tecnología } \\
\hline $\begin{array}{l}\text { Gracias a los avances tecnológicos, se obtienen } \\
\text { procesos más depurados, repetitivos y sin } \\
\text { errores ni alteraciones. Para el caso de empresas } \\
\text { industriales, los tiempos de producción se recortan } \\
\text { drásticamente. }\end{array}$ & $\begin{array}{l}\text { No todas las organizaciones se están adaptando a } \\
\text { buen ritmo a los nuevos métodos. Muchas industrias } \\
\text { corren el riesgo de quedarse muy desactualizadas en } \\
\text { poco tiempo. }\end{array}$ \\
\hline \multicolumn{2}{|l|}{ Fuerza de trabajo } \\
\hline $\begin{array}{l}\text { La posibilidad de trabajar desde distintos centros } \\
\text { de trabajo y plantas, y colaborar con equipos de } \\
\text { otros países en tiempo real. Los empleados cada } \\
\text { vez adquirirán mayores competencias para hablar } \\
\text { e interpretar el lenguaje de los datos, toma de } \\
\text { decisiones y optimizar resultados. }\end{array}$ & $\begin{array}{l}\text { La sustitución del capital humano por procesos } \\
\text { robotizados. Las empresas se ven en la necesidad de } \\
\text { prescindir de los servicios de muchas personas. }\end{array}$ \\
\hline \multicolumn{2}{|l|}{ Ámbito social } \\
\hline $\begin{array}{l}\text { Nuevas formas de comunicarse, acortar distancias, y } \\
\text { relacionarse, posiblemente se fortalezcan relaciones } \\
\text { más sanas atendiendo a políticas de bienestar social } \\
\text { no solo dentro de las mismas empresas, sino por } \\
\text { parte de los gobiernos. }\end{array}$ & $\begin{array}{l}\text { Los avances industriales a excesiva velocidad pueden } \\
\text { permitir que crezcan las desigualdades y que exista } \\
\text { cierta fragmentación social, lo cual desencadena } \\
\text { dilemas de seguridad geopolítica. }\end{array}$ \\
\hline \multicolumn{2}{|l|}{ Medio ambiente } \\
\hline $\begin{array}{l}\text { Muchas de estas tecnologías ya están ayudando a } \\
\text { resolver problemas ecológicos; esto hace pensar que } \\
\text { la sostenibilidad y el generar un medio ambiente más } \\
\text { sano es parte de la revolución. }\end{array}$ & $\begin{array}{l}\text { Teniendo en cuenta que esta industria se basa en } \\
\text { la producción desmedida de productos y servicios, } \\
\text { el ecosistema se ve afectado por las tecnologías, el } \\
\text { consumo desmedido de recursos no renovables y el } \\
\text { impacto que esto genera en el ambiente. }\end{array}$ \\
\hline \multicolumn{2}{|l|}{ Gerencia } \\
\hline $\begin{array}{l}\text { Los gerentes lograrán mayores índices de } \\
\text { productividad y sus empresas estarán en capacidad } \\
\text { de generar proyectos cada vez más creativos e } \\
\text { innovadores; podrán acceder a nuevos mercados } \\
\text { de una forma más rápida y fácil. Además, gracias a la } \\
\text { tecnología podrán tomar decisiones más acertadas } \\
\text { sobre la forma como deben manejar sus negocios. }\end{array}$ & $\begin{array}{l}\text { Los gerentes pueden llegar a reconocer los } \\
\text { cambios empresariales de esta revolución. Sin } \\
\text { embargo, algunos no estarán seguros de qué } \\
\text { hacer para beneficiar sus negocios y actividades; y } \\
\text { probablemente, muchos de los que dicen que están } \\
\text { preparados para asumir el reto, no estén tan listos. }\end{array}$ \\
\hline
\end{tabular}




\section{Relación gerencia - cuarta revolución industrial}

Entender la relación de la gerencia con la Revolución Industrial actual implica, como primera medida, reconocer que la misma no es un suceso aislado o atemporal que sorprenda al mundo; es importante tener en cuenta desde una perspectiva general, que aquello que sucede actualmente, en términos de tecnología, es la continuación de cambios generados en momentos anteriores de la historia de la humanidad.

La Revolución Industrial no cabe entenderla como un cambio súbito y radical, sino más bien como un proceso no exento de tensiones, que se fue consolidando con el transcurso de los años y tuvo en Inglaterra su referencia principal. Ese tiempo largo en su afirmación no oscureció sus consecuencias. Y es que los cambios tecnológicos introducidos por la Revolución Industrial supusieron una ruptura con el pasado mucho más drástica que cualquier otra desde la invención de la rueda. (Chaves, 2004, p. 93).

Del mismo modo debe verse la gerencia; no como un término que solo cobra importancia ahora, sino como una función que se ha desarrollado a lo largo de la historia, pero con enfoques diferentes que en su momento han atendido a las necesidades de los mercados.

Cada nuevo modelo gerencial no significa un reemplazo del existente, sino que se sustenta en el anterior, intensificándose la competitividad y ampliándose el menú de tareas para los gerentes. En fin, en el presente, la rapidez para detectar las nuevas tendencias en los patrones de conducta y necesidades de los consumidores, la velocidad para diseñar, producir y comercializar los productos para las nuevas necesidades se está convirtiendo en el modelo gerencial para las empresas líderes. (Villasmil, 2010, p. 143).

La relación que existe entre ambas variables subyace en la idea de mejorar o replantear aquello que se hace; es decir, lograr una transición hacia nuevos sistemas construidos sobre la infraestructura de la revolución digital anterior, cuyos orígenes obedecen a las primeras revoluciones industriales, como la llegada de la electrónica y la tecnología de la información y las telecomunicaciones.

\section{Conclusiones}

Finalmente, este ejercicio permite concluir que los cambios tecnológicos de la actual Revolución Industrial atienden cada vez menos a los productos, materiales y equipos; concentran su atención en cómo lograr integrar a las personas con las máquinas y los datos. La razón fundamental por la que se presenta esta inclinación está en la importancia que para las empresas puede tener la riqueza que suministran estas variables; de un lado, la creatividad e imaginación, que por naturaleza posee el ser humano y su capacidad de generar interacciones sociales para el pleno desarrollo de la vida tal como ha sido concebida. De otro lado, la aplicación artificial que sugieren las máquinas para agilizar procesos, reducir costos y mejorar las cadenas de producción. Y, finalmente, las bondades que representan los datos en cuanto a diagnósticos y resolución de problemas. 
En ese orden de ideas, el gerente contemporáneo debe estar en la capacidad de registrar, analizar y unir toda esta información de forma eficiente, buscando diseñar las estrategias de negocios futuros de la compañía. De acuerdo con las características propias de la actual revolución, se espera que las empresas sean lideradas por personas que posean una visión del todo, que puedan atender a iniciativas de transformación e innovación dejando atrás los mecanismos anteriores, a partir de tecnologías disruptivas. Capaces de preparar a sus empleados para que puedan hacer frente a los nuevos retos, a través de procesos de formación y fortalecimiento de competencias y habilidades, y que se comprometan a generar espacios de bienestar y seguridad para sus equipos de trabajo. Se espera también de los gerentes actuales y futuros, que sean competentes para medir el impacto social y ambiental de sus decisiones y poner en equilibrio el hacer el bien y el generar ganancias en el marco de la ética y la moral. De otro lado, los gerentes contemporáneos deben prestar atención a la forma en la que sus estrategias afectan el medio ambiente a partir del uso racional de los recursos naturales y las tecnologías de producción limpia.

\section{Referencias}

Álvarez, C. (2009). Innovación, competitividad y nuevos modelos gerenciales. Sinergia e Innovación, 1(08), 1-15. Recuperado de https://repositorioacademico.upc.edu.pe/bitstream/handle/10757/332789/89296-1-PB.pdf?sequence=1\&isAllowed=y

Chaves, J. (2004). Desarrollo Tecnológico en la Primera Revolución Industrial. Revista de Historia, 17. Recuperado de https://moodle2.unid.edu.mx/dts_cursos_mdl/pos/MDL/AC/AM/01/Desarrollo.pdf

Crissien, J. (2005, mayo-agosto). Gerencia del Siglo XXI. Revista Escuela de Administración de Negocios, 54, 59-83. Recuperado de https://www.redalyc.org/pdf/206/20605404.pdf

Drucker, P. (1957). La Gerencia de Empresas, $1^{\text {a }}$ edición. New York, Harper \& Brothers. Recuperado de https://www.academia.edu/31002142/Drucker_peter_-_la_gerencia_de_empresas

Drucker, P. (2002). La gerencia en la sociedad futura (Trad. Cárdenas Nannetti, J.). Bogotá, Colombia: Grupo Editorial Norma. S.A. Recuperado de http://biblio.upmx.mx/indices/151622.pdf

Fracica Naranjo, G. (2010). Gerencia Emprendedora para el Siglo XXI. Dimensión empresarial, 8 (2), 5664. Recuperado de https://dialnet.unirioja.es/descarga/articulo/3990472.pdf

Falconi Tello, L. X., López Aguirre, J. F., Pomaquero Yuquilema, J. C., \& López Salazar, J. L. (2018, julioseptiembre). Habilidades gerenciales para la revolución industrial 4.0 en el ámbito del capitalismo consciente. Revista Contribuciones a la Economía. Recuperado de https://www.eumed.net/rev/ ce/2018/3/revolucion-industrial-capitalismo.html

Ibáñez, N., \& Castillo, R. (2010, julio-octubre). Hacia una epistemología de la gerencia. Orbis. Revista Científica Ciencias Humanas, 6 (16), 54-78. Recuperado de http://www.redalyc.org/pdf/709/70916426004.pdf

Polo, G. (2012). Teoría organizacional para la gerencia humana como factor de desarrollo a escala humana. Revista Clío América, 6 (11), 52-71. Recuperado de https://revistas.unimagdalena.edu.co/index. $\mathrm{php} /$ clioamerica/article/view/425/389

La actual transición tecnológica y su relación con la gerencia empresarial 
Sallenave, J. P. (1995). La Gerencia integral. Bogotá, Colombia: Editorial Norma. Recuperado de https:// www.academia.edu/22755568/JEAN-PAUL_SALLENAVE._LA_GERENCIA_INTEGRAL

Villasmil, M. (2010). Globalización Empresarial: Desafíos del gerente en el presente. Innovación y gerencia, 2 (3), 138-155. Recuperado de https://www.researchgate.net/publication/323402185 